\title{
EMBAJADOR IGOR VELÁZQUEZ RODRÍGUEZ: SU LEGADO PERPETUO Y EJEMPLAR PARA EL PERÚ
}

\author{
Oscar Maúrtua de Romaña *
}

\section{Introducción}

Próximo a cumplirse 15 años de la penosa partida de nuestro querido amigo y colega Igor Velázquez Rodríguez (Lima, 27 de enero de 2005) y nueve años antes que la Corte Internacional de Justicia dictara el fallo sobre la controversia marítima entre Perú y Chile, debemos tenerlo siempre presente, pues fue un hombre, que, en honor al servicio a su país, adquirió y donó la sede permanente de la Academia Diplomática del Perú1; aquella, que, hasta fines del 2003, funcionaba en instalaciones alquiladas, sin que ningún miembro del Servicio Diplomático hubiese adoptado una actitud benefactora hacia el Alma Mater de la diplomacia peruana, tal cual como la asumida por Igor Velázquez Rodríguez, en una inédita acción. En reconocimiento por su importantísimo y noble gesto, se decidió nombrar a la Academia Diplomática del Perú "Embajador Igor Velázquez Rodríguez", nombre que se mantuvo hasta el 2011, esto es, por más de siete años.

La razón es elemental y existe jurisprudencia: Prevaleció la costumbre -que es fuente de Derecho- de mantener el apellido, por gratitud, de quién

\footnotetext{
* Embajador y Jurista. Ingresó a Torre Tagle a los 18 años como empleado administrativo en 1965. Graduado de la Pontificia Universidad la Católica del Perú (PUCP) como Bachiller en Humanidades. Licenciado en Derecho (Abogado) de la Universidad Nacional Mayor de San Marcos (UNMSM). Licenciado en Relaciones Internacionales de la Academia Diplomática del Perú (1968). Premio Nacional de Cultura, 1969. Postgrados de las Universidades de Oxford, John Hopkins y Comité Jurídico Interamericano de la OEA. Magíster en Gestión Pública por la Universidad Tecnológica del Perú - UTP (2018). Presidente de la Sociedad Peruana de Derecho Internacional (SPDI). Secretario General de la Presidencia de la República (1980-1985). Director de la Academia Diplomática (2002-3003) Ex Vice Ministro y Secretario General de Relaciones Exteriores; y, ex Canciller de la República. Profesor e investigador.
}

Deseo dejar expreso reconocimiento a los Embajadores Julio Balbuena López Alfaro y Heli Peláez Castro, por sus generosos aportes a la elaboración del presente ensayo.

${ }^{1}$ Bákula, Juan Miguel. (2006). El Perú en el reino ajeno. Historia interna de la acción externa. Universidad de Lima, 2004, p. 143. 
efectúa un acto de liberalidad de tipo voluntario por el cual una persona dispone de parte de su patrimonio para la transferencia de manera gratuita en favor de un tercero ${ }^{2}$, como es el caso del Instituto Riva Agüero de la Universidad Católica (PUCP), la Fundación Canevaro, el hospital Larco Herrera, la Beca Prado de la Universidad Nacional Mayor de San Marcos (UNMSM), los Premios de la Fundación Manuel J. Bustamante de la Fuente, entre otros; en qué se ha respetado el nombre del legador, ya que es meramente manifestación de reconocimiento, con contenido axiológico.

Sin embargo, el legado de este ilustre diplomático peruano va más allá de la cesión de las instalaciones en las que hoy se forman los futuros embajadores del Perú en el mundo.

Igor Velázquez Rodríguez se inició, a muy corta edad, como ayudante quinto en el Ministerio de Relaciones Exteriores, lo que refleja su sentido de identidad y de pertenencia a la institucionalidad diplomática. A partir de ese momento, su carrera no hizo más que acumular logro tras logro, llegando a ser Representante Permanente del Perú ante las Organizaciones Internacionales con sede en Viena, Austria (Agencia Internacional de Energía Atómica - O.I.E.A.; Organización de las Naciones Unidas para el Desarrollo Industrial - O.N.U.D.I.; Comisión Preparatoria de la Organización para la Prohibición Total de Ensayos Nucleares, y; Acuerdo Regional de Cooperación para la Promoción de la Ciencia y la Tecnología Nuclear en América Latina y el Caribe - ARCAL) y Embajador del Perú en países de Europa Central.

Considerado como un diplomático audaz, agudo, visionario, con capacidad para la negociación, pero sobre todo para anteponerse a distintos tipos de situaciones, con valentía y con claridad de ideas, no cabe duda de que Igor Velázquez Rodríguez supo defender y promover los intereses del país y llevar el nombre del Perú en todo lo alto, en el ejercicio de sus funciones diplomáticas y humanas.

Más aún, en un país como el nuestro, donde no abundan los actos de filantropía y en tiempos en que la autosuficiencia y el egoísmo marcan el

\footnotetext{
${ }^{2}$ Estos son los sustentos de la donación. El autor consultó diversas fuentes, entre ellas: - Ghersi, Carlos A.; Weingarten, Celia (2017). "Contrato de donación». Manual de contratos civiles, comerciales y de consumo. Ciudad Autónoma de Buenos Aires: La Ley. p. 269. ISBN 978-987-03-3194-0.

- Castillo Freyre, Mario, "Tratado de los Contratos Típicos", vol. XIX, tomo 1, cap. La donación, PUCP, Fondo Editorial, 2002, pág. 165.
} 
devenir humano, acciones como la suya sobresalen, cuando no generan sorpresa, ante tan infrecuente desapego y altruismo.

\section{Sus inicios}

Igor Velázquez Rodríguez, nacido en Madrid el 25 de marzo de 1933, fue hijo del reconocido escritor y poeta piurano, Juan Luis Velázquez Guerrero (Piura, 26 de julio de 1903) ${ }^{3}$, así como de doña Dolores Rodríguez Zubillaga (Madrid, 3 de junio de 1914), notable empresaria y relacionista pública española. La pareja se separó a los pocos años de matrimonio y ella se asentó en el Perú. ${ }^{4}$

Sobre el padre de Igor, Juan Luis Velázquez Guerrero, cabe destacar que fue educado en el colegio la Inmaculada (SJ) en Lima y llegó a ser Doctor en Letras por la Universidad Nacional Mayor de San Marcos, siendo su primer poemario vanguardista El perfil de frente (1924), encomiado por Luis Alberto Sánchez, Jorge Basadre y José Carlos Mariátegui. Asimismo, mantuvo estrecha amistad con César Vallejo durante sus años en España y París, y fue amigo y colaborador cercano de Trotsky en México.

Mirko Lauer calificó a Juan Luis Velázquez, como “incuestionable autor" apasionado de la síntesis, la misma que refleja en su primer libro: "El Perfil de Frente", obra que luego de aparecer, le hizo preguntarse ¿Puede haber algo más revelador en la búsqueda de la síntesis victoriosa, de la unidad reconquistada, que ese título? ${ }^{5}$

Agrega Raúl Moscol León, en Juan Luis, 31 años después: "Y es que los versos que forman parte de este poemario son breves. Unos tienen nombre, otros no y al final, el bardo ha escrito el mes y año de su inspiración, como el siguiente que data de noviembre del 23:

Ya me diste todo quedando integra y pura.

\footnotetext{
${ }^{3}$ Milla Batres, Carlos (2004). Diccionario Biográfico del Perú Contemporáneo. Siglo $X X$. Tomo II (N-Z). Editorial Milla Batres, Lima, p. 442.

4 Enciso, Mary. (2015). Evocando al inolvidable Igor Velázquez. En "El Mirador”. Publicado el 3 de febrero de 2015.

Página electrónica: https://elmirador.lamula.pe/2015/02/03/evocando-al-inolvidableigor-velasquez/encisopress/

${ }^{5}$ Raúl F. Moscol León. (2001). Juan Luis, 31 años después. Artículo publicado en el Diario Correo de Piura, el 25 de agosto de 2001. Ver en Literatura Piurana: http://rafemole-literaturapiurana.blogspot.com/2008/12/juan-luis-velazquez-31-aosdespues.html
} 
Jamás perderé, aunque te pierda, la eternidad de tu dulzura.

Ya no serás, como lo fuiste, mía y tuyo". 6

Moscol también nos relata que "Juan Luis admiraba a Albert Einstein. Lo llamó Héroe Contemporáneo. La Editora Libro-Mex de México publicó un libro que escribió sobre el creador de la Teoría de la Relatividad. También se nutrió de Buda, Guyau y Trotsky y pese a la gran amistad que cultivó con el líder soviético que vivió y fue asesinado en México, se alejó de los trotskistas 'cuando se disfrazaron como los únicos poseedores de una verdad mal digerida y peor administrada y mi comunismo halló su fin cuando lo vi vestirse de policía secreta'. [...] Y después de sentirse incapaz de 'vivir una fe militante y exclusivista', el poeta se convirtió en pintor 'porque sólo la pintura es capaz de decir todos los matices dentro de la armonía. Es el único arte unitario'. Optó, además, por el silencio y supo que era cierto aquello de 'Qué soledad sin soledad siquiera', versos que llevan el nombre de 'Piura', borroneados en marzo de 1924, que recuerdan a la "gente tan llena de recodos/enlodados en este desierto sin lluvias / ni rastrojos.",7

Cabe indicar, además, que la hermana de Igor, Sonia Velázquez Rodríguez, se casó con el Embajador Jaime Cacho Souza Castro. Son sus sobrinos carnales de Igor, los hijos de dicha unión, los actuales Embajadores Jaime y Manuel Cacho Souza Velázquez.

Igor Velázquez Rodríguez, realizó sus estudios escolares primarios en el Colegio Anglo-Peruano (Perú) y los secundarios en el Liceo FrancoMexicano (México) y en el Colegio La Salle (Perú). Heredó de sus padres una exigente sintaxis y una pulcra redacción, con sindéresis y fino uso gramatical. Hablaba perfectamente inglés, francés, alemán, checo, húngaro $\mathrm{y}$ varios otros idiomas.

Asimismo, finalizando la secundaria, Igor Velázquez Rodríguez decidió ser médico, por lo que optó por entrar a San Fernando, la facultad de Medicina de la Universidad Nacional Mayor de San Marcos - UNMSM. No obstante, no pudo finalizar sus estudios, los cuales solo duraron dos años, debido a que dicha casa de estudios fue clausurada por razones políticas.

\footnotetext{
${ }^{6}$ Ibídem.

${ }^{7}$ Ibídem.
} 


\section{De su trayectoria diplomática}

Luego del cierre de la Facultad de Medicina de San Marcos, es el momento en donde se inicia la inmersión de Igor en el Servicio Diplomático del Perú. Julio Salazar, su íntimo amigo, hace un recuento de cómo fue el verdadero inicio de su vida diplomática, señalando que su padrastro, médico de cabecera del Presidente del Perú en la época, el General Manuel A. Odría, teniendo en consideración el mensaje que le había transmitido su señora esposa Doña Dolores Rodríguez, sobre las aspiraciones que tenía su menor hijo Igor para ser diplomático, se lo comunicó al Jefe de Estado, obteniendo el padrastro, una respuesta positiva. ${ }^{8}$

De esta manera es que Odría llamó al Canciller interino de la época, Eduardo Miranda Sousa, titular del despacho del Fomento, para que se agende la incorporación del joven Igor Velázquez Rodríguez al Ministerio de Relaciones Exteriores. Sin embargo, transcurrió un prudencial periodo de tiempo, tres meses, y la llamada nunca se hizo realidad. Al parecer la negativa del Canciller para hacer caso al pedido del Presidente Odría, se sustentaba en la corta edad que tenía Igor en ese entonces, la cual no sobrepasaba los 20 años. A insistencias del Presidente, es que Igor Velázquez, por fin, hace su entrada al Ministerio de Relaciones Exteriores, como ayudante quinto, el 17 de setiembre de 1952, estando de Canciller, don Ricardo Rivera Schreiber; y, el 20 de julio de 1953, se inscribe en el Escalafón del Servicio Diplomático del Perú.

Es así que se dio inicio a la larga trayectoria diplomática de Igor Velázquez Rodríguez: Tercer Secretario, mediante R.S. N $^{\circ} 312$ del 20 de julio de 1953; Segundo Secretario, a través de la R.S. N ${ }^{\circ} 287$ del 1 de abril de 1961; Primer Secretario, por R.S. N ${ }^{\circ} 56$ del 28 de enero de 1966; Consejero, mediante R.S. $N^{\circ} 0001$ del 1 de enero de 1970; Ministro, a través de la R.S. No 0001 del 1 de enero de 1973; y, Embajador, por R.S. N $^{\circ} 0703$ del 9 de diciembre de 1977.

Debido a su agudeza, perspicacia y dotes para la negociación, cualidades imprescindibles para un diplomático, Igor fue asumiendo altas funciones y responsabilidades -luego de varios años de servicio- como "Encargado de Negocios a.i. del Perú" en Estados Unidos y en Chile. Uno de sus más destacados logros, desempeñando sus funciones en Washington D.C., fue impedir que se le aplicaran al Perú "Enmiendas" (represalias económicas que portan los nombres de los Congresistas norteamericanos que

8 Salazar, Julio. (2008). En Recuerdo de un amigo. En revista "Política Internacional", revista de la Academia Diplomática del Perú. Lima 2008. 
las proponen y que afectan la asistencia estadounidense en una Nación que según ellos perjudica los intereses de E.E.U.U.) llevadas a cabo por el gobierno norteamericano, tales como la Hickenlooper, Pelly, Comte y la PL 480, sustentándose en las expropiaciones que había realizado el gobierno militar del Perú en los años 70's; asimismo, evitó que atuneros californianos violaran la soberanía de las 200 millas del Perú. ${ }^{9}$

Cuando ascendió a la categoría de Embajador, Igor Velázquez, quien se preocupaba constantemente por el futuro institucional, y siendo Director de Personal de la Cancillería, se empeñó en encontrar una solución al grado profesional que nos otorgaba la Academia Diplomática, que era de "Diplomático de Carrera". Tras diversas y persistentes gestiones ante la Asamblea Nacional de Rectores (ANR), logró que se otorgue el título de Licenciado en Relaciones Internacionales, promoviendo legalmente que tal formalización académica se confiera también, con efecto retroactivo, a las generaciones precedentes.

También puedo dar testimonio de su caballerosidad con el Presidente Fernando Belaunde y su esposa Violeta Correa de Belaunde, cuando éste se hallaba exiliado en Washington D.C., modales democráticos y de respeto que no practicaron otros representantes diplomáticos peruanos en los Estados Unidos.

Asimismo, ejerció funciones como Embajador del Perú en cuatro países de Europa central, siendo estos, Hungría, Polonia, Checoslovaquia y Austria (donde simultáneamente se desempeñó como Embajador no Residente del Perú ante el Gobierno de Turquía).

Posteriormente, fue designado Representante Permanente del Perú ante los Organismos Internacionales con sede en Viena, siendo también Presidente del Grupo Latinoamericano y del Caribe (GRULAC), en los que desempeñó una extraordinaria labor en personificación de los intereses del Perú. Admiraba a Cancilleres -con quienes había colaborado de cerca- como Carlos García Bedoya, José de la Puente Rabdill, Arturo García y García; así como a Embajadores como Jorge Guillermo Llosa Pautrat, Luis Marchand Stens, Carlos Alzamora, Fernando Berckemeyer y Celso Pastor de La Torre.

No cabe duda, que Igor Velázquez Rodríguez fue una de las figuras más representativas de la diplomacia peruana, dejando en claro las habilidades imprescindibles para desempeñar las funciones que amerita ser

9 Enciso, Mary. Ibídem 
miembro del Servicio Diplomático: patriotismo; inteligencia; honradez; lealtad; elegante talante; entre otras virtudes.

\section{Legado y reconocimientos}

Es de conocimiento público, que, hasta fines del 2003, la Academia Diplomática del Perú, no contaba con un local propio. La realidad cambió a inicios del 2004 cuando Igor Velázquez Rodríguez, en reconocimiento al honor de haber servido a su Patria, decide ex profesamente comprar y ceder las acciones y derechos de las instalaciones de un inmueble ubicado en San Isidro (esquina de Av. Pershing $\mathrm{N}^{\circ} 335$ y calle Ugarte y Moscoso $\left.\mathrm{N}^{\circ} 101\right)^{10}$, para ser la actual sede permanente de la Academia Diplomática del Perú. Fui testigo de ese ejemplar gesto, por encontrarme como Director de dicha institución de formación profesional. De esta manera, Igor permitía que los futuros representantes del país puedan forjarse académicamente en instalaciones idóneas y propias para con las funciones que la Academia lleva a cabo. Esta acción, es un legado invaluable, propio de un hombre con valores y principios, pero sobre todo con un amor manifiesto por su patria y por la institución que lo acogió y a la que consagró más de medio siglo de su vida.

En agradecimiento a la importante donación, las Asociaciones de Funcionarios Diplomáticos del Perú (AFSDP), en actividad (AFDA) y en retiro (AFDR) -esto es, todo el Servicio Diplomático-, manifestaron su concordancia de que la Academia Diplomática del Perú lleve su nombre. Entonces, por disposición de la Resolución Ministerial N ${ }^{\circ}$ 0435/RE del 4 de junio de $2004^{11}$, se agradece la donación de una sede permanente y se resuelve que la Academia Diplomática del Perú lleve el nombre de "Igor Velázquez Rodríguez". ${ }^{12}$

Posteriormente, esta decisión de denominar a la Academia Diplomática "Igor Velázquez Rodríguez", fue ratificada mediante

${ }^{10}$ Dicha donación fue anunciada mediante Carta del 24 de marzo de 2004, dirigida al entonces Ministro de Relaciones Exteriores, Embajador Manuel Rodríguez Cuadros.

${ }^{11}$ Modificada en su séptimo Considerando por la Resolución Ministerial No 0496/RE del 30 de junio de 2004.

${ }^{12}$ También es sabido que el Presidente del Consejo de Ministros, Armando Villanueva del Campo en más de una oportunidad le ofreció ser nombrado como Viceministro Secretario General de Relaciones Exteriores, esto es Jefe Servicio Diplomático de la Republica, lo que Igor Velázquez no se decidió en aceptar, recordando el pensamiento hegeliano:" ... de que sea yo bueno y los demás mejores que yo", lo que reafirma su vocación institucional y la sencillez de su alma. 
Resolución Suprema N ${ }^{\circ}$ 297-2005-RE del 24 de noviembre de 2005, la cual lleva la firma del Presidente Constitucional de la República y del suscrito, como Ministro de Estado en el despacho de Relaciones Exteriores. Asimismo, el Gobierno ya le había concedido la Orden del Sol del Perú, en el grado de Gran Cruz, el 25 de junio de 2004, como reconocimiento a su trayectoria profesional. ${ }^{13}$

A pesar de estos títulos y reconocimientos, en el 2011, siete años más tarde, a iniciativa del Embajador Harry Belevan $\mathrm{McBride}^{14}$, el Presidente de la República, Alan García Pérez, junto con el Canciller José Antonio García Belaunde, poco antes de terminar su mandato, deciden, mediante Resolución Suprema $\mathrm{N}^{\circ} 136-2011-\mathrm{RE}$ del 20 de abril de 2011, modificar el nombre de la Academia Diplomática del Perú, que, desde entonces, llevaría el nombre del ilustre diplomático, ex Secretario General de las Naciones Unidas, "Javier Pérez de Cuéllar".

Naturalmente, la decisión acarreó un sinfín de opiniones diversas, a favor y en contra del cambio de nombre, creando desazón y frustración entre quienes habíamos contribuido a reconocer tan plausible identidad con nuestra Alma Mater. El bien inmueble prevaleció con la denominación de "Casa Embajador Igor Velázquez Rodríguez".

Sin embargo, es imprescindible nunca olvidar al hombre que le donó una sede permanente y con prestancia a la Academia Diplomática del Perú, quien no lo hizo por buscar algún reconocimiento, sino por la honra y gratitud de haber podido servir a su Patria, incluso después de su muerte. En tal tesitura, Mery Enciso en su artículo "Evocando al inolvidable Igor Velázquez"15, cita las nobles y elocuentes palabras - que aún perduran- del

${ }^{13}$ Cabe resaltar que mucho antes, a fines de la década de los 70's, el Ministro de Relaciones Exteriores, Embajador Carlos García Bedoya, lo había nombrado Presidente de su Comité de Asesores y luego fue condecorado con la Orden "Al Mérito por Servicios Distinguidos en Grado de Gran Cruz" (RS. Nº041 del 17 de enero de 1979). Asimismo, a nivel internacional, recibió las siguientes condecoraciones: Orden “Bernardo O’Higgins" en el Grado de Gran Cruz - Chile (1979); Orden "Del Águila Azteca” en el grado de Placa - México (1980); y, Orden "De Mayo Al Mérito" en el Grado de Gran Oficial - Argentina (1980), entre otras.

14 Según él mismo lo ha afirmado en una entrevista para el diario La República, el 5 de marzo de 2020, la que está disponible en: https://larepublica.pe/politica/2020/03/06/javier-perez-de-cuellar-soy-un-productode-torre-tagle-quimicamente-puro/

${ }^{15}$ Enciso, Mary. (2015). Evocando al inolvidable Igor Velázquez. En “El Mirador”. Publicado el 3 de febrero de 2015. 
ex Canciller Manuel Rodríguez Cuadros en su sepelio: "Él fue un diplomático por vocación, por función, por identidad. Una suerte de diplomático ontológico en el sentido que siempre asumió su misión personal, familiar y como ciudadano a partir de su desempeño como Embajador del Perú y como miembro del Servicio Diplomático".

Todo esto coincide y se engarza con la doctrina de Abraham Maslow ${ }^{16}$ quien en su pirámide considera en la cima de la misma, la autorrealización; es decir, el honor.

Su filantropía no se limitó a su donativo para la sede de la Academia Diplomática, sino fue también en memoria a su madre, doña Dolores Rodríguez Zubillaga (16-02-1993) que donó, igualmente, una fuente en el corazón de San Isidro, en la intersección de las avenidas Miguel Dasso y Cavenecia, en homenaje a la madre peruana; a la vez que aportó discretamente sendas subvenciones a viudas de colegas diplomáticos que no atravesaban buena situación financiera. Similar filantropía realizó con la construcción de una sede en Chilca para acoger a personas humildes. Lo revelo porque han habido trascendidos, ya que a él no le hubiera agradado que fuera de conocimiento público dado su fino talante.

Estoy persuadido, que el amor de Igor por el Servicio Diplomático y su personalidad, encajan perfectamente en el concepto español de caballero y que en el vocablo ingles se expresa como un "gentleman"; esto es, el hombre valeroso que no daña, que no perjudica a nadie, que no odia, sino que se consagra por sus valores, y señorío. Y esa es la estela que nos deja este entrañable, inteligente, desinteresado amigo y colega a quien recordamos constantemente.

Todos los años, hace ya quince, los amigos leales a Igor Velázquez Rodríguez, llevamos a cabo una romería al cementerio donde descansan sus restos y nos congregamos en una cofradía para evocar al proverbial donante con afecto, respeto y admiración, seguros que su personalidad icónica trasciende para las nuevas generaciones de peruanos, que deben conocer de su desprendimiento y grandeza humana, ya que se ha convertido Igor en leyenda de la memoria colectiva del Perú.

Enero de 2020.

Página electrónica: https://elmirador.lamula.pe/2015/02/03/evocando-al-inolvidableigor-velasquez/encisopress/

${ }^{16}$ Maslow, Abraham. (1973). A Theory of Human Motivation. Página electrónica: https://books.google.es/books?id=vdvXOxzbiNwC\&hl=es 


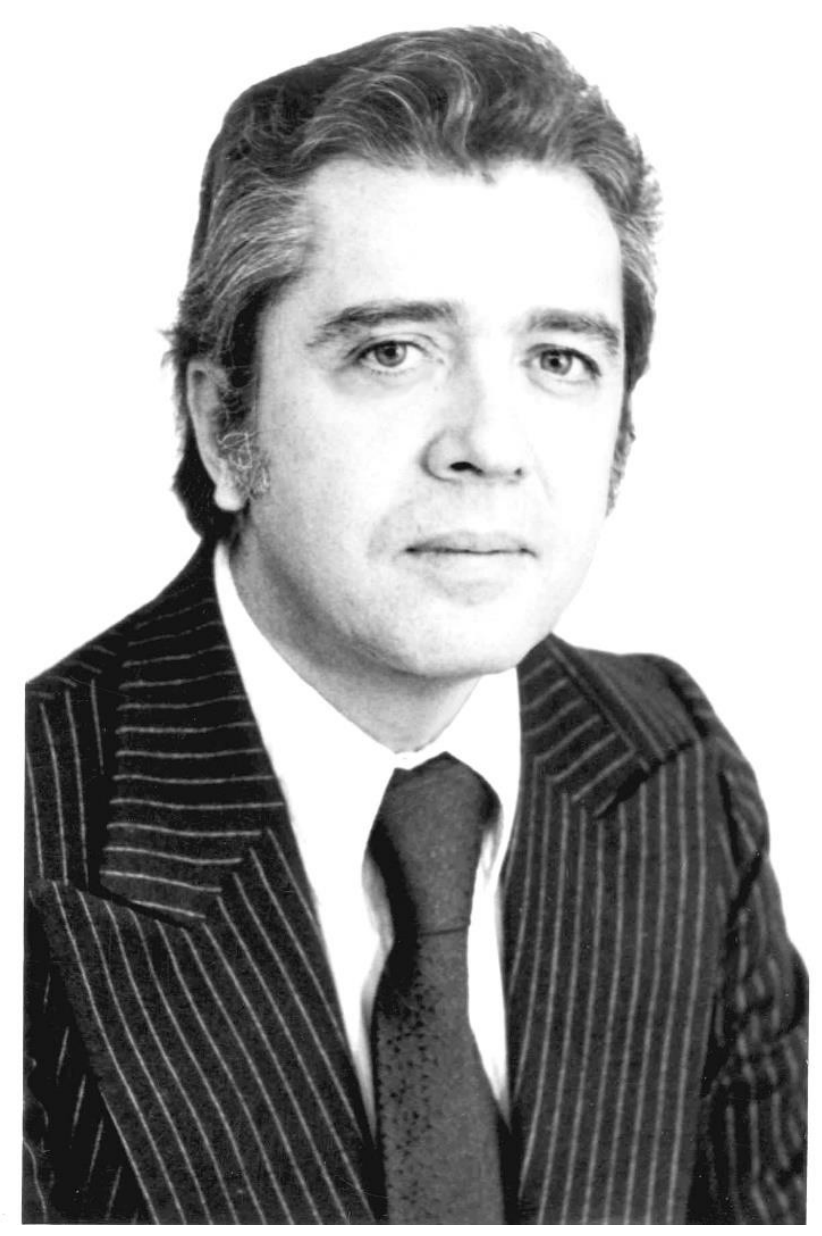

\section{Igor Velázquez Rodríguez}

(Madrid 1933 - Lima 2005) 


\section{BIBLIOGRAFÍA}

Bákula, Juan Miguel. (2006). El Perú en el reino ajeno. Historia interna de la acción externa. Universidad de Lima, 2004, p. 143.

Castillo Freyre, Mario, “Tratado de los Contratos Típicos”, vol. XIX, tomo 1, cap. La donación, PUCP, Fondo Editorial, 2002, pág. 165.

Enciso, Mary. (2015). Evocando al inolvidable Igor Velázquez. En "El Mirador”. Publicado el 3 de febrero de 2015. Página electrónica: https://elmirador.lamula.pe/2015/02/03/evocando-al-inolvidable-igorvelasquez/encisopress/

Ghersi, Carlos A.; Weingarten, Celia (2017). «Contrato de donación». Manual de contratos civiles, comerciales y de consumo. Ciudad Autónoma de Buenos Aires: La Ley. p. 269. ISBN 978-987-03-3194-0.

Maslow, Abraham. (1973). A Theory of Human Motivation. Página electrónica: https://books.google.es/books?id=vdvXOxzbiNwC\&hl=es

Milla Batres, Carlos (2004). Diccionario Biográfico del Perú Contemporáneo. Siglo XX. Tomo II (N-Z). Editorial Milla Batres, Lima, p. 442.

Moscol León, Raúl F. (2001). Juan Luis, 31 años después. Artículo publicado en el Diario Correo de Piura, el 25 de agosto de 2001. Ver en Literatura Piurana: http://rafemole-literaturapiurana.blogspot.com/2008/12/juan-luis-velazquez-31aos-despues.html

Resolución Ministerial Nº 0496/RE del 30 de junio de 2004.

Salazar, Julio. (2008). En Recuerdo de un amigo. En revista "Política Internacional", revista de la Academia Diplomática del Perú. Lima 2008.

El presente texto se elaboró en ocasión del 15 aniversario del fallecimiento de Igor Velázquez Rodríguez y que fue impreso para tal oportunidad. 